\title{
Flood Detection Using Multi-Modal and Multi-Temporal Images: A Comparative Study
}

\author{
Kazi Aminul Islam ${ }^{1}$, Mohammad Shahab Uddin ${ }^{1}$, Chiman Kwan ${ }^{2}$ (D) and Jiang Li ${ }^{1, *(1)}$ \\ 1 Department of Electrical \& Computer Engineering, Old Dominion University, Norfolk, VA 23529, USA; \\ kisla001@odu.edu (K.A.I.); muddi003@odu.edu (M.S.U.) \\ 2 Applied Research LLC, Rockville, MD 20850, USA; chiman.kwan@arllc.net \\ * Correspondence: jli@odu.edu
}

Received: 29 June 2020; Accepted: 28 July 2020; Published: 30 July 2020

\begin{abstract}
Natural disasters such as flooding can severely affect human life and property. To provide rescue through an emergency response team, we need an accurate flooding assessment of the affected area after the event. Traditionally, it requires a lot of human resources to obtain an accurate estimation of a flooded area. In this paper, we compared several traditional machine-learning approaches for flood detection including multi-layer perceptron (MLP), support vector machine (SVM), deep convolutional neural network (DCNN) with recent domain adaptation-based approaches, based on a multi-modal and multi-temporal image dataset. Specifically, we used SPOT-5 and RADAR images from the flood event that occurred in November 2000 in Gloucester, UK. Experimental results show that the domain adaptation-based approach, semi-supervised domain adaptation (SSDA) with 20 labeled data samples, achieved slightly better values of the area under the precision-recall (PR) curve (AUC) of 0.9173 and F1 score of 0.8846 than those by traditional machine approaches. However, SSDA required much less labor for ground-truth labeling and should be recommended in practice.
\end{abstract}

Keywords: flood detection; deep convolutional neural network; domain adaptation; SVM; MLP

\section{Introduction}

Flooding is one of the most severe forms of natural disasters. It affected all over the world and caused millions of human deaths [1,2]. Flooding is the second deadliest natural disaster in the United States and hurricanes Katrina, Mathews, Florence and Sandy caused severe property damages and thousands of lives were lost [3]. To mitigate the damage of flooding, an early accurate estimation of the flooded area is a necessary step for a rescue team to help the people in the affected area.

Throughout the years, researchers developed automatic methods to monitor flood events using remote sensing data. Automatic methods are quick, cost-effective, and monitoring from distance as compared to traditional methods, which require labor, in-person visit and longer processing time. In remote sensing domain, wide range of sensors are available. Some provide better spatial resolutions and others provide more frequent temporal revisit. Remote sensing-based flood-detection methods can be divided into two categories: optical and radar sensor. Optical data such as WorldView2 [4], Landsat [5-7], Spot-5 [8], sentinel [9], Moderate-Resolution Imaging Spectroradiometer (MODIS) [7,10] and RADAR [8,11-13] had been explored for flood detection. In the meanwhile, machine-learning algorithms including decision tree algorithm [4,14], SVM [6,8], MLP [8] had been developed for flood detection.

The data fusion technical committee (DFTC) of the IEEE Geo-Science and Remote Sensing Society organized a contest to perform flood detection using a multi-temporal and multi-modal dataset in 2009 [8]. This dataset contains SPOT-5 satellite images and radar images from both the pre- and post-flooding event occurred in 2000 in Gloucester, UK. In the contest, traditional machine-learning 
methods including SVM and MLP were winners in the supervised flood-detection scenario. The SVM model used the SPOT-5 satellite images combined with four derived morphological channels from the near infrared (NIR) channel in the original data for flood detection. The MLP model used both the SPOT-5 satellite and radar images. Both the MLP and SVM methods concatenated the pre- and post-event flooding data in training and testing and the two models provided similar performance [8]. Liu et al. introduced a homogeneous pixel transformation algorithm [15] to detect flood using the same DFTC dataset [8].

Deep Learning is a subset of machine-learning techniques. We called the machine-learning model as a deep learning model when we use multiple layers of neural network. Deep learning model has been used in wide range of applications including computer vision [16-18], speech-based medical diagnosis [19,20], cybersecurity [21,22], medical disease diagnosis $[19,23]$, remote sensing $[17,24,25]$ domains. To train a deep learning model from a scratch, we usually need a lot of labeled data because deep learning models contain a large number of trainable parameters. This usually posts a challenge in practice because it is not always possible to collect sufficient labeled data for training. To solve this problem, one possible solution is to fine-tune a pre-trained model with limited available labeled data. Examples can be found in biomedical application [26], facial expression recognition [27] and plant recognition [28].

Flood detection attracted significant amount of attention after the competition and new datasets and deep learning algorithms had been developed for this purpose. Deep learning algorithms such as deep neural network [29], deep convolutional neural network (DCNN) [30,31] and fully convolutional neural networks (F-CNNs) [32] with satellite images including Sentinel-2 [30], Planet [31], Landsat [32] and Google Earth aerial imagery [29] have been used for flood detection. Unmanned aerial vehicles (UAV) image data with VGG-based fully convolutional network (FCN-16s) was also developed for flood detection [33].

Most of the flood-detection methods including the winners of the competition concatenated both the pre- and post-event images for training [8]. For example, the ground-truth of region of interest (ROI) in the competition were defined by examining both the pre- and post-event images and flood and non-flooded regions were identified as ground-truth ROIs for training. However, it is not always possible to obtain ground truth after the flood event in practice, making the developed methods challenging to be applied in practice.

An alternative is to train a model using ROIs defined on pre-event images and directly apply the trained model to post-event images for flood detection. However, models trained in one domain usually cannot perform well in another domain if there are data distribution shifts between the two domains, and there is a need to incorporate domain adaptation techniques to address the distribution shift challenge. For example, if we train a classifier using the ground truths defined in pre-event images and use the trained classifier directly to detect flood in post-event images, our experimental results on the competition dataset showed that the performances dropped significantly.

To address this issue, researchers have developed many domain adaptation approaches among which the generative adversarial network (GAN) [34]-based approaches have been very popular for image classification and segmentation. Tzeng et al. used a GAN loss to mimic the target domain to represent the source domain [35]. Motiian et al. used a Siamese architecture where they used a few samples to adapt a model into different domain [36]. Our previous study used a semi-supervised domain adaptation approach for seagrass detection and achieved better results in seagrass detection as compared with other methods [18].

The motivation of this study is to perform a comparative study on the various domain adaptation algorithms with those competition winners for flood detection on the competition dataset, and provide useful practical suggestions for flood detection. Contributions of this paper are:

- We implemented domain adaptation methods and compared these methods with traditional machine-learning algorithms for flood detection. To the best of our knowledge, we are the first to implement a deep learning-based domain adaptation approach for flood detection. 
- Our experimental results showed that domain adaptation-based methods can achieve competitive performance for flood detection and require much less labeled samples in the post-event images for model fine-tuning.

- Our recommendation for the community is that domain adaptation methods require less labor and are better tools for flood detection.

The paper is structured as follows: Section 2 describes data and experiment design. Sections 3 and 4 present results and discussions, respectively, and Section 5 summarizes the paper.

\section{Dataset and Experiment Design}

\subsection{Dataset}

We used two heterogeneous satellite image sensors including SPOT-5 and a European Remote Sensing 1 (ERS-1) SAR images as shown in Figure 1 from the competition [8]. The images were captured from the flooding event occurred in November 2000 in Gloucester, UK. The SPOT-5 optical sensor image contains three bands including Red, Green, and Near-inferred-1 and radar image only contains the SAR channel. The green, red and near IR channels have spectral ranges of $0.50-0.60 \mu \mathrm{m}$, 0.61-0.68 $\mu \mathrm{m}$, and $0.78-0.89 \mu \mathrm{m}$, respectively, with $20 \mathrm{~m}$ spatial resolution. The SAR image uses C-band, $5 \mathrm{GHZ}$ frequency with linear VV polarization. The SAR images also use wave mode to collect the temporal images with a spatial resolution of $10 \mathrm{~m}$ and a swath width of $5 \mathrm{~km}$.

The pre-event and post-event images are shown in Figure 1. Two temporal images of SPOT-5 are taken in September 2000 and November 2000 as shown in Figure 1a,c. The pre-event and post-event of SAR images are taken in October 2000 and November 2000 as displayed in Figure 1b,d, respectively. The original ground truth provided by the organizers were found to be inaccurate and we used the updated ground truth [15] in our study as shown in Figure 2b. For the SAR image, we took a logarithm operation on the pixel values and applied a de-speckle filtering process with a $7 \times 7$ window to improve its resolution.
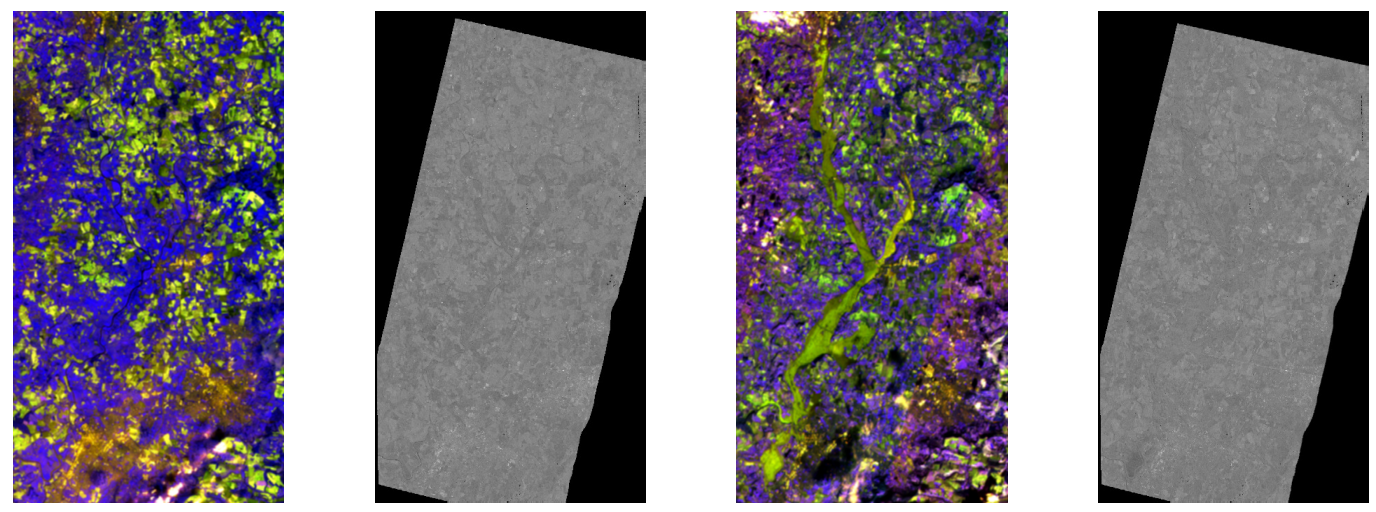

(a) Pre-event SPOT image (b) Pre-event SAR Image (c) Post-event SPOT Image (d) Post-event SAR Image

Figure 1. Multi-modal and multi-temporal Images taken in Gloucester, UK. Spot-5 images are displayed using Green, Red and NiR Band.

\subsection{Data Augmentation with Morphological Operation}

Some winners of the competition augmented the data with morphological operation. For a fair comparison, we also created four additional bands using morphological operation as described in [8]. We used opening by reconstruction (OR) operation on the NIR band with a circular structuring element radius of 40 and 100. In addition, we implemented closing by reconstruction (CR) operation on the images resulted from the last step with a circular structuring element radius of 60 and 90 . In total, 
we created four additional bands based on the original images. The created four bands for the pre-event and post-event image are shown in Figure 3.

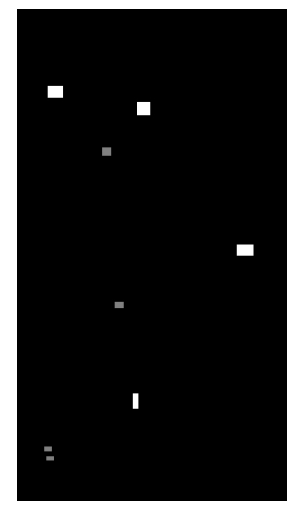

(a)

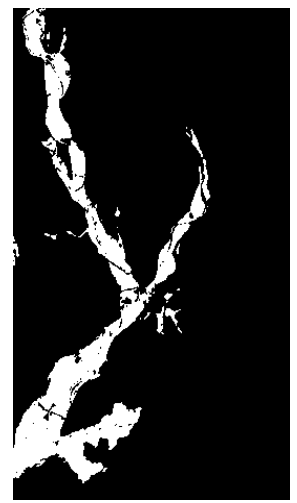

(b)

Figure 2. Ground Truth. (a) Selected ROIs in the pre-event image; (b) Updated flooding ground truth in the post-event image [15].

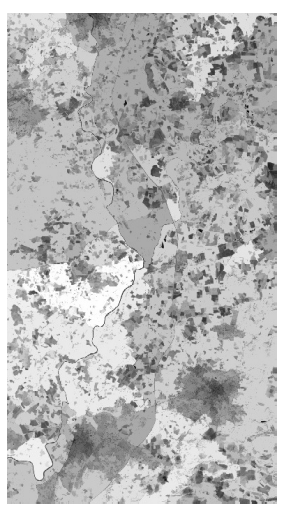

(a) OR 40

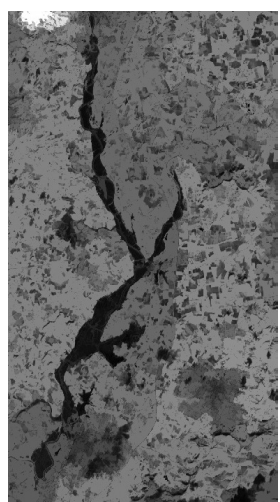

(e) OR 40

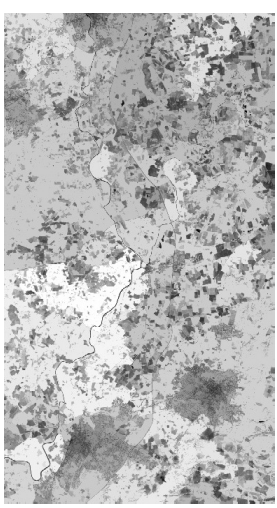

(b) CR 60

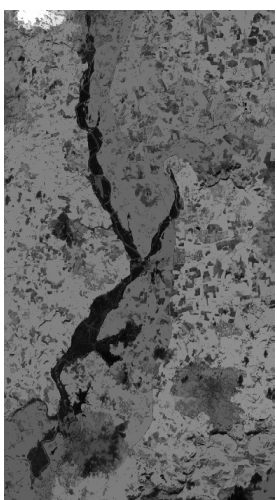

(f) CR 60

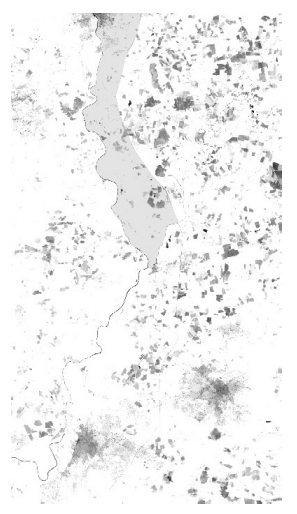

(c) OR 100

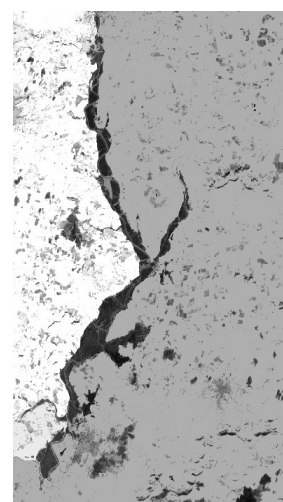

(g) OR 100

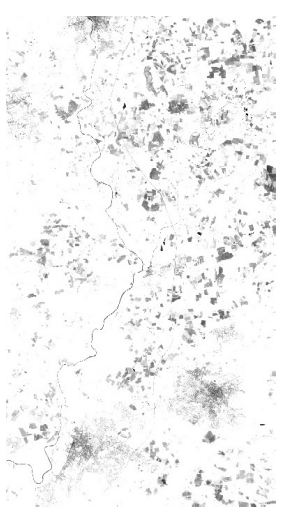

(d) CR 90

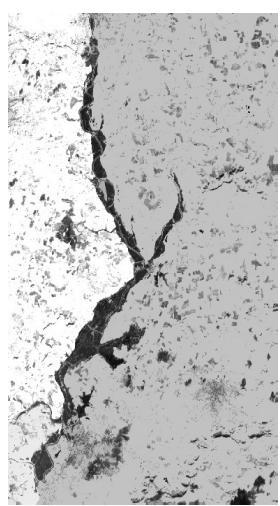

(h) CR 90

Figure 3. Derived morphological bands based on the pre-event (1st row) and post-event (2nd row) SPOT-5 image's NIR band. CR: Closing by Reconstruction. OR: Opening by Reconstruction. 


\subsection{Models for Comparison}

\subsubsection{Multi-Layer Perceptron (MLP)}

Multi-layer Perceptron (MLP) is also known as feed-forward artificial neural network (ANN) uses a perceptron algorithm to perform tasks. Geoffrey Hinton et al. introduced a back-propagation algorithm [37] which made the MLP algorithm effective and widely popular among researchers. The back-propagation algorithm adjusts the weights of the MLP model to minimize the difference between the actual output and the desired output [37]. MLP usually uses three layers of network to perform classification and regression tasks. MLP algorithm has been effectively used for flood detection by the winners of the competition [8].

We re-implemented the MLP classifier that achieved the best performance in the data fusion contest [8]. This model stacked the SPOT-5 and radar images from both the pre-event $(t 1)$ and post-event (t2) for flood detection. The stacked data consists of 8 bands as $\left[X_{R}^{t_{1}}, X_{G}^{t_{1}}, X_{N I R}^{t_{1}}, X_{S A R}^{t_{1}}, X_{R}^{t_{2}}\right.$, $\left.X_{G}^{t_{2}}, X_{N I R}^{t_{2}}, X_{S A R}^{t_{2}}\right]$. The model was trained using the labeled regions of interest (ROIs) in both the $t_{1}$ and $t_{2}$ images and then was applied to the whole images for flood detection. The architecture of the MLP model is shown in Figure 4.

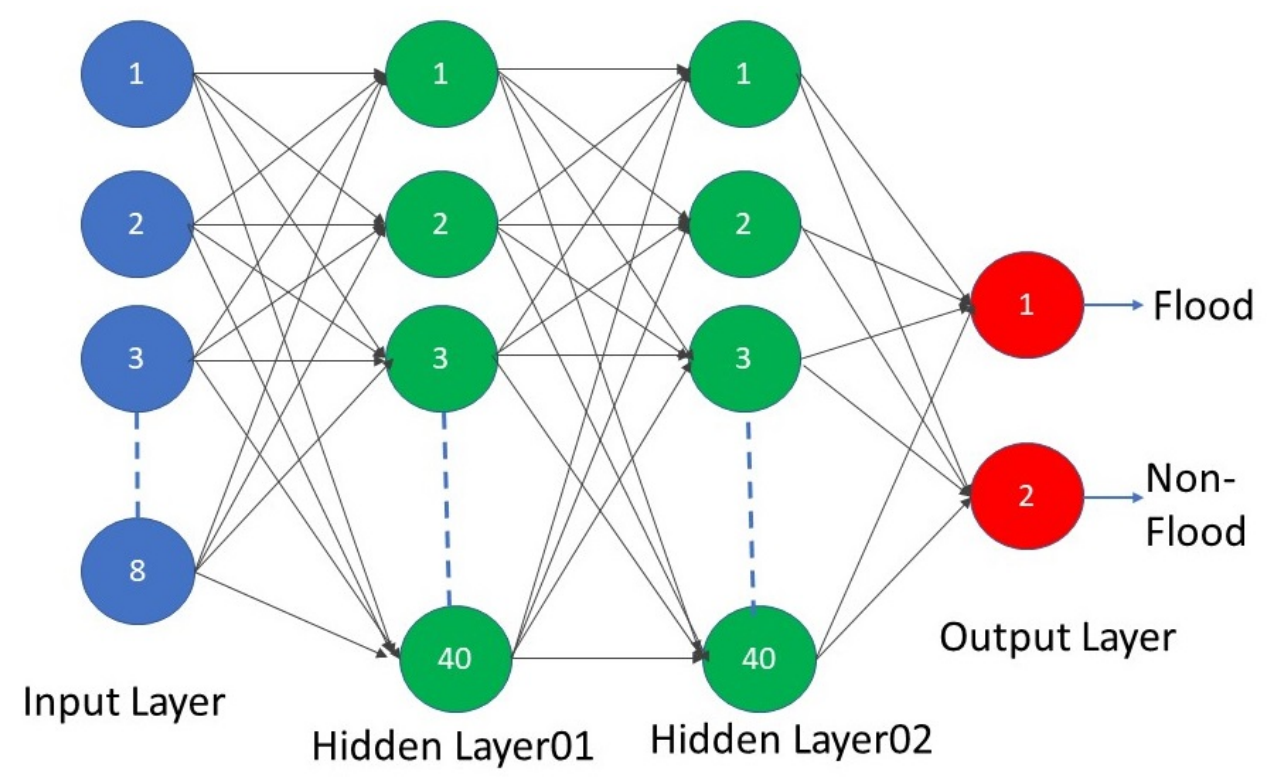

Figure 4. Multi-layer Perceptron Architecture.

\subsubsection{Support Vector Machine (SVM)}

Support Vector Machine [38] is another popular machine-learning method which has been widely used for classification and is considered to be a baseline for image classification. SVM finds hyper-planes among data points as classification boundaries. Linear, polynomial, Gaussian radial basis function, and Hyperbolic tangent kernel is generally used for SVM classification. LIBSVM [39] is a popular implementation for SVM models.

We followed the same settings of another winner in the competition who used an SVM classifier for flood detection [8]. This model used the four additional bands created by morphological operation on the NIR band in the post-event's SPOT image as described in Section 2.2, making the input data 10 bands as $\left[X_{R}^{t_{1}}, X_{G}^{t_{1}}, X_{N I R}^{t_{1}}, X_{R}^{t_{2}}, X_{G}^{t_{2}}, X_{N I R}^{t_{2}}, X_{N I R O R 40}^{t_{2}}, X_{N I R O R 100}^{t_{2}}, X_{N I R C R 60}^{t_{2}}, X_{N I R C R 90}^{t_{2}}\right]$. Similarly, the model was trained using the labeled ROIs in both the $t_{1}$ and $t_{2}$ images and then was applied to the whole images for flood detection. 


\subsubsection{Source Only (SO)}

To compare with the domain adaptation-based flood-detection methods, we treated the pre-event images as source domain and the post-event as target domain. The SO method is the baseline model, in which we trained a classifier (CNN/MLP/SVM) only using the ROIs labeled in the pre-event images. We then directly applied the trained classifier to the post-event images for flood detection.

In source only models, we only used the pre-event image's ROIs to learn the difference between the flood and non-flood data without using any labeled and unlabeled data from the post-event images. The CNN-SO model's architecture is shown in Figure 5. Here, the DCNN model has two convolutional layers and two fully connected layers. The convolutional layers use filters to search for task-specific features in the images. If it finds similar features present in the images, the filters produce a high agreement. Initially, the filters are random but after a few iterations, the filters are optimized to scan task-specific features. After two convolutional layers, we used a fully connected layer as shown in Figure 5. The fully connected layer works similarly to the MLP model. The last layer performs the classification task and predicts the probability of being flood class or non-flood class.

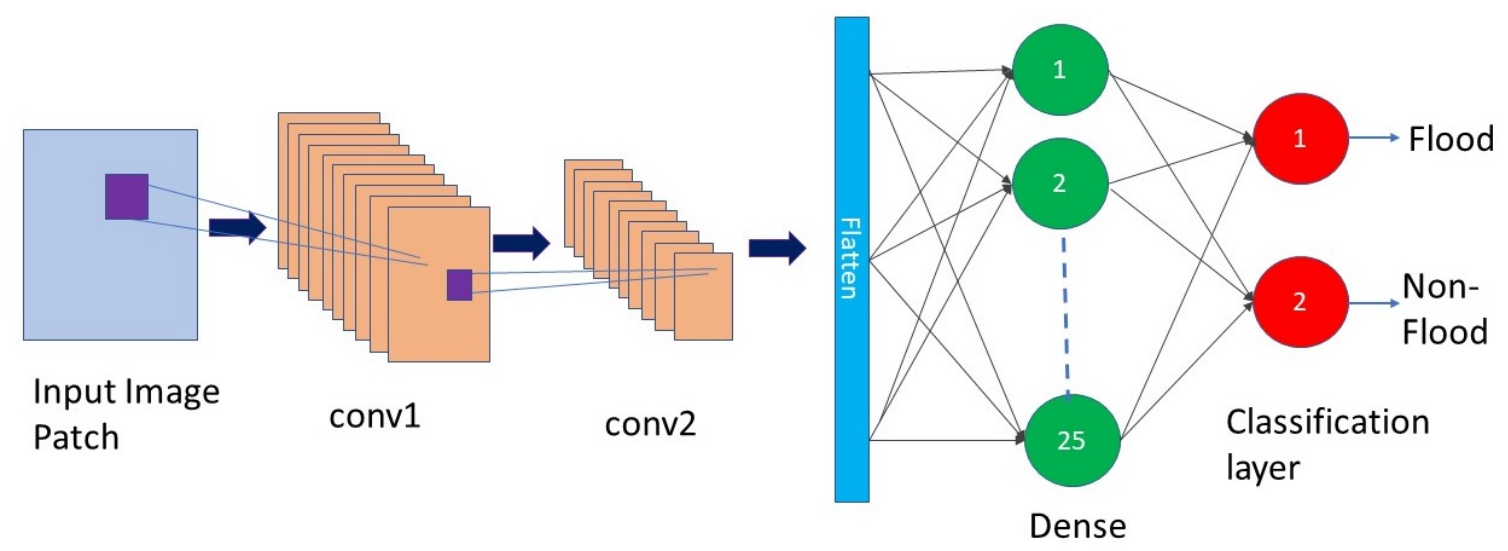

Figure 5. Deep Convolutional Neural Network Architecture.

We stacked the images as $\left[X_{R}^{t 1}, X_{G}^{t 1}, X_{N I R}^{t 1}, X_{S A R}^{t 1}, X_{N I R O R 40}^{t 1}, X_{N I R O R 100}^{t 1}, X_{N I R C R 60}^{t 1}, X_{N I R}^{t 1}{ }_{N R 90}\right]$ for training, and $\left[X_{R}^{t 2}, X_{G}^{t 2}, X_{N I R}^{t 2}, X_{S A R}^{t 2}, X_{N I R}^{t 2}\right.$ OR40 $, X_{N I R O R 100}^{t 2}, X_{N I R}^{t 2}{ }_{N 60}, X_{N I R}^{t 2}$ CR90 $]$ for flood detection. We normalized the data between 0 to 1 in each band and used the labeled ROIs in the pre-event images for training, no label information in the post-event images were used during training. All subsequent methods described in Sections 2.3.4-2.3.7 used the same data composition.

\subsubsection{Unsupervised Domain Adaptation (UDA)}

Unsupervised domain adaptation based on generative adversarial network (GAN) [35] became popular since GAN was created in 2014 [34], and we implemented the approach proposed for flood detection. First, we trained a deep convolutional neural network (DCNN) model in the source domain using all the labeled ROIs. After the training, the DCNN model learned the source embedding function, $G_{S}$ and source classifier $C_{s}$ for flood detection (Figure 6a). In the second step, we used the unlabeled data from both the source and target domains to match the marginal distribution between the source $\left(P\left(G_{S}\left(X^{s}\right)\right)\right)$ and the target domains $\left(P\left(G_{t}\left(X^{t}\right)\right)\right)$ based on the GAN loss (Figure $6 \mathrm{~b}$ ). The target embedding was trained to represent the source embedding so that the classifier trained on the source domain was used in the target domain for flood detection.

Let the source and target domain datasets to be denoted as $D_{s}=\left\{X^{s}, Y^{s}\right\}$ and $D_{t}=\left\{X^{t}\right\}$, respectively. First, we train a DCNN model using the labeled samples from the source domain (Figure 6a),

$$
L_{c}\left(f_{s}\right)=E\left[l\left(f_{s}\left(X^{s}\right), Y^{s}\right)\right]
$$


where $f_{S}$ is the DCNN model and $E$ is the expectation function and $l$ is any related loss functions. $f_{s}$ can be divided into two function source embedding function, $G_{s}$ and source classifier, $C_{s}$. We use the trained source embedding function $G_{s}$ to find an optimum target embedding function $G_{t}$ using the unlabeled data from both domains. We modify the target domain $G_{t}$ using a GAN loss to match the source domain embedding function $G_{s}$. The discriminator, $D$, in Figure $6 \mathrm{~b}$ is trained using following GAN loss,

$$
L_{a d v_{D}}\left(X^{s}, X^{t}, G_{s}, G_{t}\right)=E_{x^{s} \sim X^{s}}\left[\log D\left(G_{s}\left(x^{s}\right)\right)\right]-E_{x^{t} \sim X^{t}}\left[\log \left(1-D\left(G_{t}\left(x^{t}\right)\right)\right)\right]
$$

The target embedding function $G_{t}$ uses the following generator loss to mimic the source domain,

$$
\operatorname{Min}_{G_{t}} L_{a d v_{G}}\left(X^{s}, X^{t}, D\right)=-E_{x^{t} \sim X^{t}}\left[\log D\left(G_{t}\left(x^{t}\right)\right)\right]
$$

Using this feedback, the target embedding function $G_{t}$ modifies its parameter so that the source domain classifier can work on the target domain.

\subsubsection{Semi-Supervised Domain Adaptation (SSDA)}

We modified our previous work [18] for flood detection as shown in Figure 6. First, a DCNN model was trained using the source domain labeled ROIs. After training, the source embedding function was first adapted to target domain using UDA described in Section 2.3.4. Then, a few labeled samples from the target domain were used to align the class-specific distribution between the source and the target domain based on the semantic contrastive alignment and classification loss (CSA) (Figure 6c). The CSA loss put the same class samples from different domains together and different class samples from the different domains as far as possible (Figure 6d).

To reduce the classwise distribution shift between the source and target domain, we used a few labeled samples from the target domain $D_{t}=\left\{X^{t}, Y^{t}\right\}$ with the classification and contrastive semantic alignment loss (CCSA) [18,36]. The classification loss was defined as,

$$
L_{C}(G \circ C)=E[l(f(X), Y)]
$$

The contrastive semantic alignment (CSA) loss consists of semantic alignment loss and class separation loss,

$$
L_{C S A}(G)=L_{S A}\left(G_{t}\right)+L_{C S}\left(G_{t}\right)
$$

where $L_{S A}\left(G_{t}\right)$ is the semantic alignment loss and $L_{C S}\left(G_{t}\right)$ is the class separation loss. $L_{S A}\left(G_{t}\right)$ is computed as,

$$
L_{S A}\left(G_{t}\right)=\sum_{a=1}^{N_{c}} d\left(p\left(G_{S}\left(X_{a}^{s}\right)\right), p\left(G_{t}\left(X_{a}^{t}\right)\right)\right)
$$

where $N_{c}$ is the number of classes, $X_{a}^{s}=X^{s} /\{Y=a\}$ and $X_{a}^{t}=X^{t} /\{Y=a\}$ are conditional random variables and $d$ is a distance metric between source $X_{a}^{s}$ and target $X_{a}^{t}$ distributions.

We used the semantic alignment loss to map samples from the same class from source domain and target as close as possible. We also used the following class separation loss to map samples carrying different class labels from different domains as far as possible in the embedding space,

$$
L_{C S}\left(G_{t}\right)=\sum_{a, b \mid a \neq b} k\left(p\left(G_{s}\left(X_{a}^{s}\right)\right), p\left(G_{t}\left(X_{b}^{t}\right)\right)\right)
$$

where $k$ denotes the similarity matrix. If the distributions of $X_{a}^{s}$ and $X_{b}^{t}$ are close to each other the loss function puts a penalty to keep them separate. Figure $6 \mathrm{~d}$ represents the working procedure of the CSA loss. The semantic alignment, class separation and classification loss are represented as the orange arrows, red dashed line and blue solid line, respectively. During testing, we used the trained 
target mapping function, $G_{t}$, to find the new representation. The overall classification and contrastive semantic alignment loss becomes,

$$
L_{C C S A}\left(G_{t}\right)=L_{C}\left(G_{t} \circ C_{t}\right)+L_{S A}\left(G_{t}\right)+L_{C S}\left(G_{t}\right)
$$

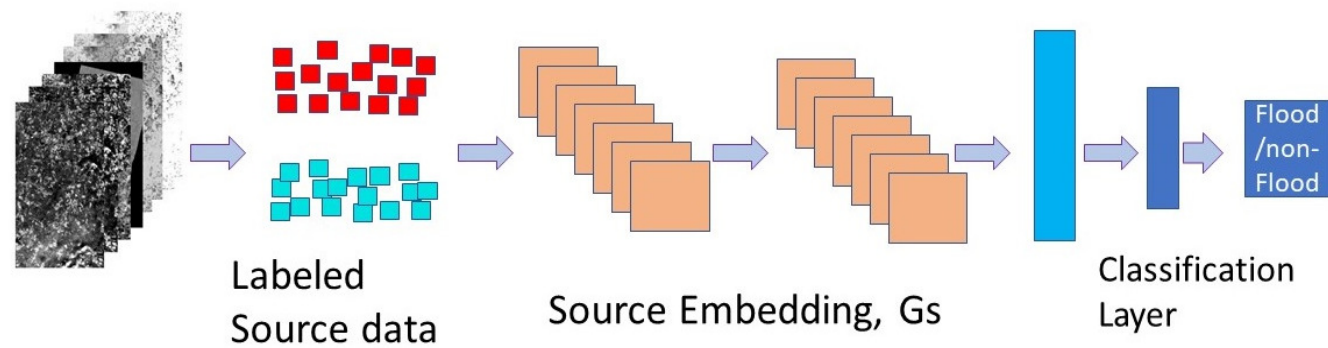

(a) Source domain supervised DCNN model training

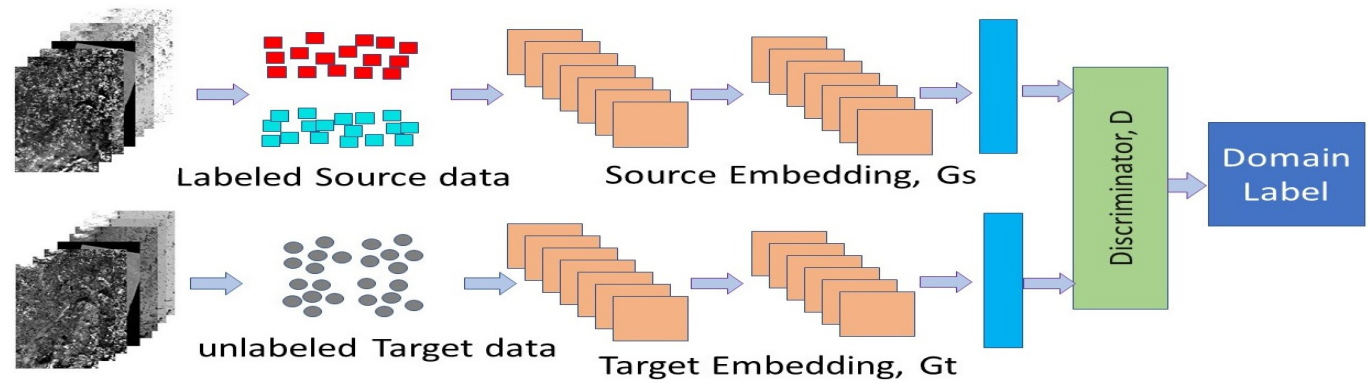

(b) Target domain embedding function learned using unsupervised adversarial domain adaptation

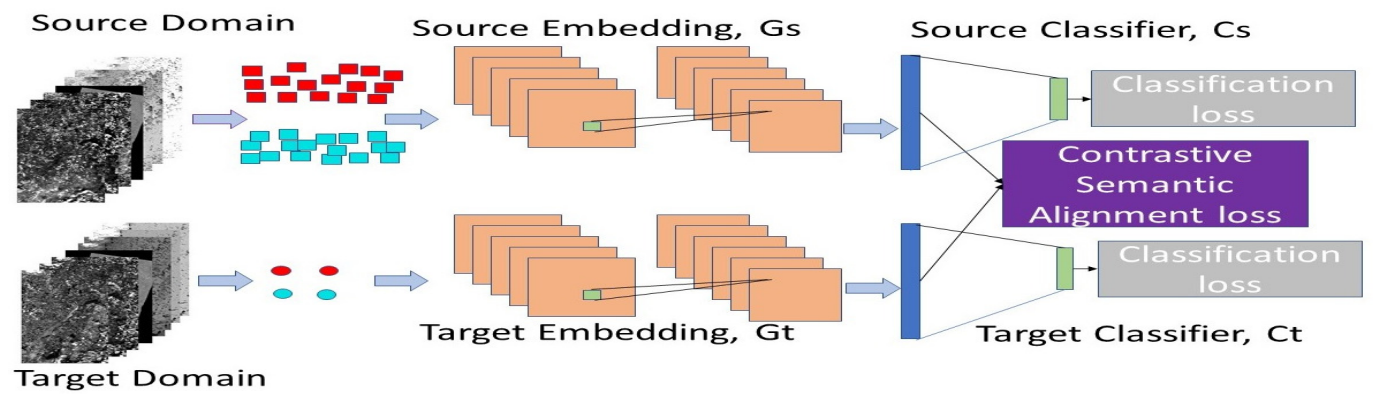

(c) Target domain supervised adaptation using CCSA loss
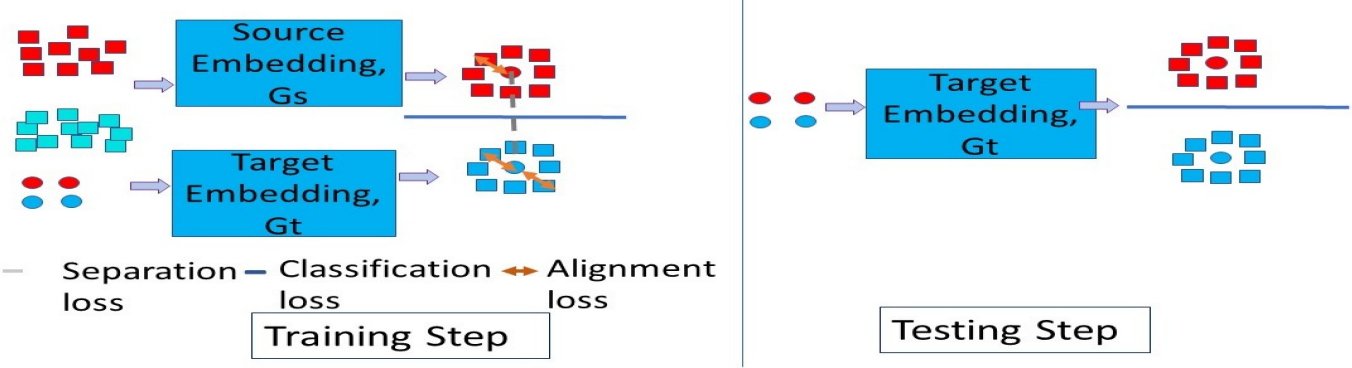

(d) Training and testing steps of CCSA loss using labeled samples

Figure 6. Semi-supervised domain adaptation (SSDA) method for flood detection. 
2.3.6. SVM, MLP and DCNN Fine-Tuned with 1, 3, 5, 10, and 20 Samples/Shots

First, we used the labeled ROIs in the pre-event images to train the classifiers (SVM/MLP/DCNN). Then, we used 1, 3, 5, and 20 labeled samples from the post-event images to fine-tune the classifiers. The fine-tuned classifiers were applied to classify the post-event images.

\subsubsection{MLP and SVM with 20 Samples from Post-Event Images}

We combined the ROIs in the pre-event images with 20 labeled samples from ROIs in the post-event images to train the SVM and the MLP classifiers. After training, we used trained classifiers for flood detection. In other domain adaptation methods, the pre-event image ROIs were used for training and a few labeled samples from post-event images were used to adapt the trained model to detect flood in target domain. This experiment was conducted to test if the sequential use of pre- and post-event labeled samples can provide any advantage.

\subsection{Evaluation Metrics}

Our dataset contains much more non-flooded pixels than flooded ones and is highly imbalanced. It was found that for binary imbalanced dataset, the precision-recall (PR) curve is a good performance metric to assess effectiveness of different classification models $[40,41]$. The area under the PR curve (AUC) is also computed as a performance metric. In addition, we apply 0.5 as threshold to flood-detection probability maps to obtain the final detections, which are used to compute precision, recall and F1 score metrics for different models as follows.

$$
\begin{gathered}
\text { Precision }=\frac{\# \text { of true flood pixels detected }}{\text { \# of total pixels detected }} \\
\text { Recall }=\frac{\# \text { of true flood pixels detected }}{\# \text { of total labeled flood pixels }} \\
F 1-\text { score }=\frac{2 * \text { Precision } * \text { Recall }}{\text { Precision }+ \text { Recall }}
\end{gathered}
$$

\subsection{Hyper-Parameter Determination}

We selected a patch-size of $5 \times 5$ in our experiments after considering the trade-off between performance and computational time. In the SSDA method, 10,000 labeled samples from each class in the source domain were randomly selected to pair with a few labeled samples in the target domain for adaptation. For the embedding functions in source domain, $G_{s}$ and target domain, $G_{t}$, we used two convolutional layers followed by a flatten layer as embedding functions. The first layer had 25 filters with a size of $2 \times 2 \times 8$ and the second layer had 25 filters with a size of $4 \times 4 \times 20$. All layers used the ReLu activation function. Both classifiers, $C_{s}$ and $C_{t}$ in $\mathrm{CNN}$ models contained a fully connected layer with 25 hidden units. The output layer had 2 units with SoftMax activation function for classification. We trained the source CNN models for 200 epochs with a batch size of 128. We trained the UDA step for 300 epochs and the SSDA step for 240 epochs in all experiments. We followed similar settings of the competition [8] and used an SVM classifier from the scikit-learn library [42] with a linear kernel. The SVM was implemented based on libsvm [39]. We use a grid search optimization technique to optimize the SVM classifier's parameters. We perform a grid search among linear and RBF kernels, $C$ parameters between 1 to 1000 and Gamma value between $1 \times 10^{-3}$ and $1 \times 10^{-4}$. Similarly, the MLP classifier had two hidden layers with 40 hidden unites each.

\section{Results}

In Table 1, the classification performances of SVM source only (SO), MLP SO, and DCNN SO methods are shown in the first three rows. The unsupervised domain adaptation results are shown in the fourth row in Table 1. Row 5 to row 23 are results by 1-shot, 3-shot, 5-shot, 10-shot, and 20-shot 
from the post-event images with MLP fine-tuning (FT), SVM FT, DCNN FT, and Semi-supervised domain adaptation (SSDA) approaches. The last two rows show results by the two winners: MLP [8] and SVM [8] in the flood-detection competition.

Table 1. Performance comparison. Precision, Recall, and F-1 score are calculated using 0.5 as threshold, and the area under the PR curve (AUC) values are computed on the OPP and MO-DS images. OPP: Original predication probability. MO-DS: Morphological operation with a disk structure.

\begin{tabular}{|c|c|c|c|c|c|c|c|c|}
\hline \multirow{2}{*}{ Methods } & \multicolumn{2}{|c|}{ Recall } & \multicolumn{2}{|c|}{ Precision } & \multicolumn{2}{|c|}{ F1-Score } & \multicolumn{2}{|c|}{ PR-AUC } \\
\hline & OPP & MO-DS & OPP & MO-DS & OPP & MO-DS & OPP & MO-DS \\
\hline SVM-SO & 0.9761 & 0.9740 & 0.1950 & 0.2108 & 0.3251 & 0.3466 & 0.7904 & 0.8132 \\
\hline MLP-SO & 0 & 0 & 0 & 0 & NA & NA & 0.2032 & 0.2203 \\
\hline CNN-SO & 0.8853 & 0.8571 & 0.3653 & 0.4201 & 0.5172 & 0.5638 & 0.7681 & 0.8070 \\
\hline UDA & 0.8764 & 0.8554 & 0.8246 & 0.8754 & 0.8497 & 0.8652 & 0.8508 & 0.8833 \\
\hline MLP 20 shots & 0.9007 & 0.8593 & 0.2017 & 0.2206 & 0.3295 & 0.3510 & 0.2460 & 0.2605 \\
\hline DCNN 1-shot FT & 0.8076 & 0.7757 & 0.5683 & 0.6201 & 0.6671 & 0.6892 & 0.8281 & 0.8524 \\
\hline MLP 1-shot FT & 0.9311 & 0.9279 & 0.4871 & 0.5305 & 0.6396 & 0.6751 & 0.7900 & 0.8617 \\
\hline SVM 1-shot FT & 0.0723 & 0.0654 & 0.0115 & 0.0105 & 0.0199 & 0.0181 & 0.0485 & 0.0484 \\
\hline SSDA 1-shot & 0.8407 & 0.8111 & 0.8623 & 0.8972 & 0.8513 & 0.8520 & 0.7155 & 0.8433 \\
\hline DCNN 3-shot FT & 0.8699 & 0.8514 & 0.7772 & 0.8259 & 0.8209 & 0.8385 & 0.7783 & 0.8115 \\
\hline MLP 3-shot FT & 0.9168 & 0.9118 & 0.6045 & 0.6539 & 0.7286 & 0.7616 & 0.8470 & 0.8829 \\
\hline SVM 3-shot FT & 0.9092 & 0.9023 & 0.7182 & 0.7631 & 0.8025 & 0.8269 & 0.8780 & 0.8969 \\
\hline SSDA 3-shot & 0.9341 & 0.9306 & 0.5409 & 0.5899 & 0.6851 & 0.7221 & 0.8906 & 0.9062 \\
\hline DCNN 5-shot FT & 0.7934 & 0.7600 & 0.8975 & 0.9261 & 0.8422 & 0.8349 & 0.8349 & 0.8577 \\
\hline MLP 5-shot FT & 0.8404 & 0.8199 & 0.8765 & 0.9055 & 0.8580 & 0.8605 & 0.8910 & 0.9064 \\
\hline SVM 5-shot FT & 0.8792 & 0.8650 & 0.8244 & 0.8614 & 0.8509 & 0.8632 & 0.8797 & 0.8984 \\
\hline SSDA 5-shot & 0.8493 & 0.8216 & 0.8989 & 0.9293 & 0.8734 & 0.8721 & 0.8209 & 0.8901 \\
\hline DCNN 10-shot FT & 0.8349 & 0.8088 & 0.8635 & 0.9004 & 0.8490 & 0.8521 & 0.8163 & 0.8439 \\
\hline MLP 10-shot FT & 0.8505 & 0.8313 & 0.8515 & 0.8862 & 0.8510 & 0.8578 & 0.8813 & 0.9019 \\
\hline SVM 10-shot FT & 0.8720 & 0.8562 & 0.8460 & 0.8798 & 0.8588 & 0.8678 & 0.8820 & 0.8998 \\
\hline SSDA 10-shot & 0.8750 & 0.8518 & 0.8515 & 0.9027 & 0.8631 & 0.8765 & 0.8506 & 0.9035 \\
\hline DCNN 20-shot FT & 0.8812 & 0.8619 & 0.7472 & 0.8317 & 0.8087 & 0.8466 & 0.8663 & 0.8925 \\
\hline MLP 20-shot FT & 0.8756 & 0.8502 & 0.7879 & 0.8465 & 0.8294 & 0.8484 & 0.8194 & 0.8966 \\
\hline SVM 20-shot FT & 0.8901 & 0.8704 & 0.8012 & 0.8566 & 0.8433 & 0.8634 & 0.8532 & 0.8960 \\
\hline SSDA 20-shot & 0.8831 & 0.8704 & 0.8681 & 0.8992 & 0.8755 & 0.8846 & 0.8669 & 0.9173 \\
\hline SVM [8] & 0.9393 & 0.9333 & 0.6263 & 0.7126 & 0.7515 & 0.8082 & 0.8442 & 0.8727 \\
\hline MLP [8] & 0.9406 & 0.9287 & 0.5472 & 0.7139 & 0.6919 & 0.8073 & 0.8567 & 0.8949 \\
\hline
\end{tabular}

\section{Original Predicted Probability (OPP) Results}

Each method produced a flooding probability map and we computed Precision, Recall and F1-score on the thresholded original maps for comparison, shown as 'OPP' in Table 1. We also compare the PR-AUC values to evaluate the flood-detection performance of each method. The OPP maps for different models are shown in Figure 7. We use a 'jet' color-map (Matlab) to show the flood-detection probability for each method. Color blue represents the probability ' 0 ' and color red represents probability ' 1 '. Also, we add a color-bar to display different probability values. The PR curves for the last six flood-detection scenarios in Table 1 are shown in Figure 8a. 


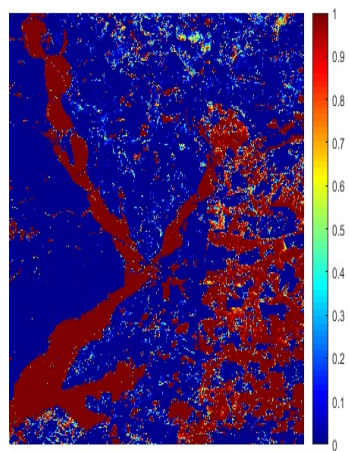

(a) $\mathrm{CNN}-\mathrm{SO}$

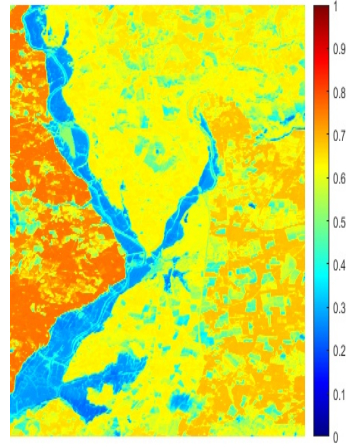

(e) SVM 1-shot FT

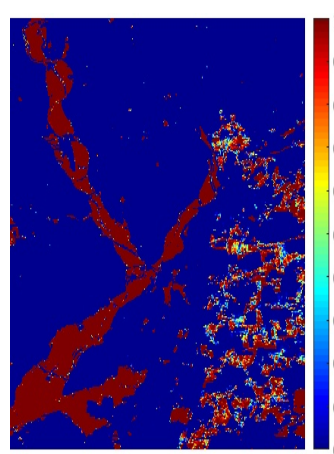

(i) DCNN 1-shot FT

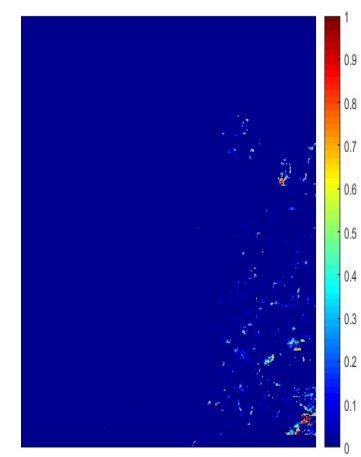

(b) MLP SO

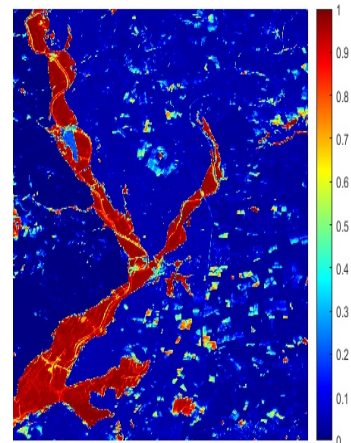

(f) SVM 20-shot FT

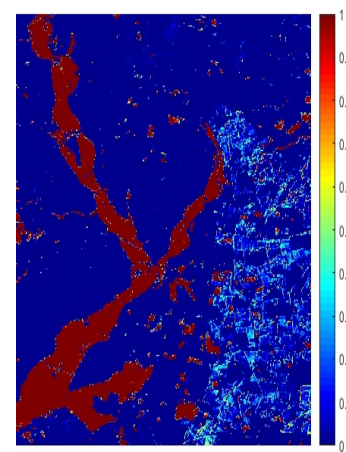

(j) DCNN 20-shot FT

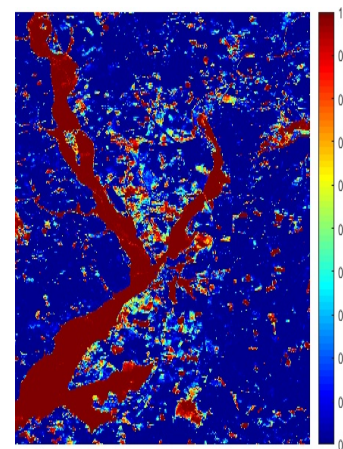

(m) SVM [8]

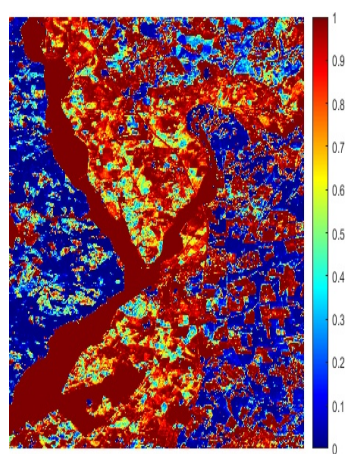

(c) SVM-SO

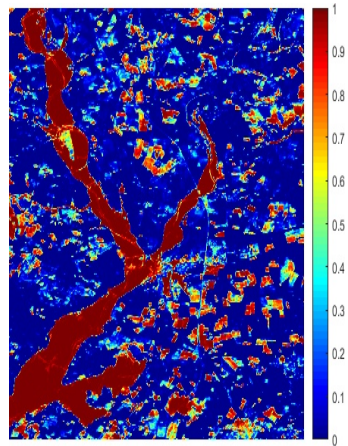

(g) MLP 1-shot FT

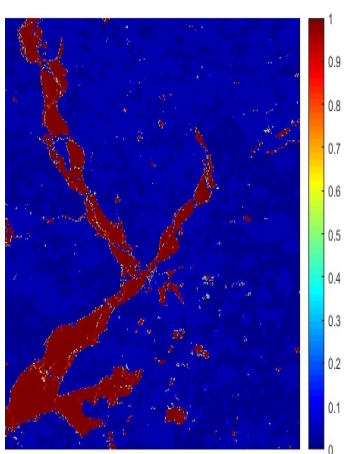

(k) SSDA 1-shot

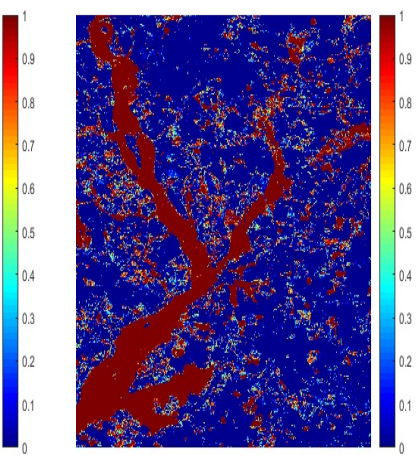

(n) MLP [8]

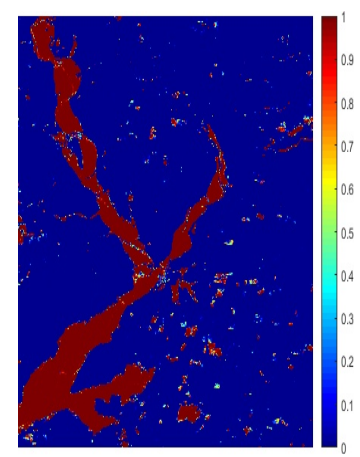

(d) UDA

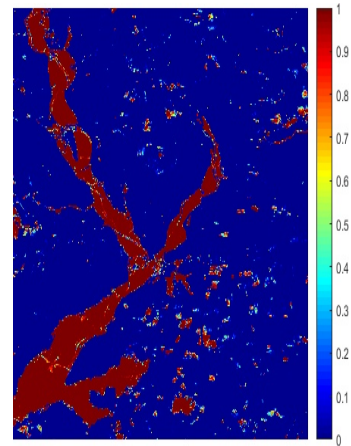

(h) MLP 20-shot FT

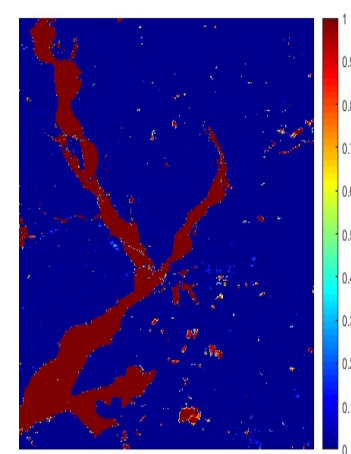

(1) SSDA 20-shot

Figure 7. Original predicted probability (OPP) maps. Blue represents the probability ' 0 ' and Red represents probability ' 1 '. 


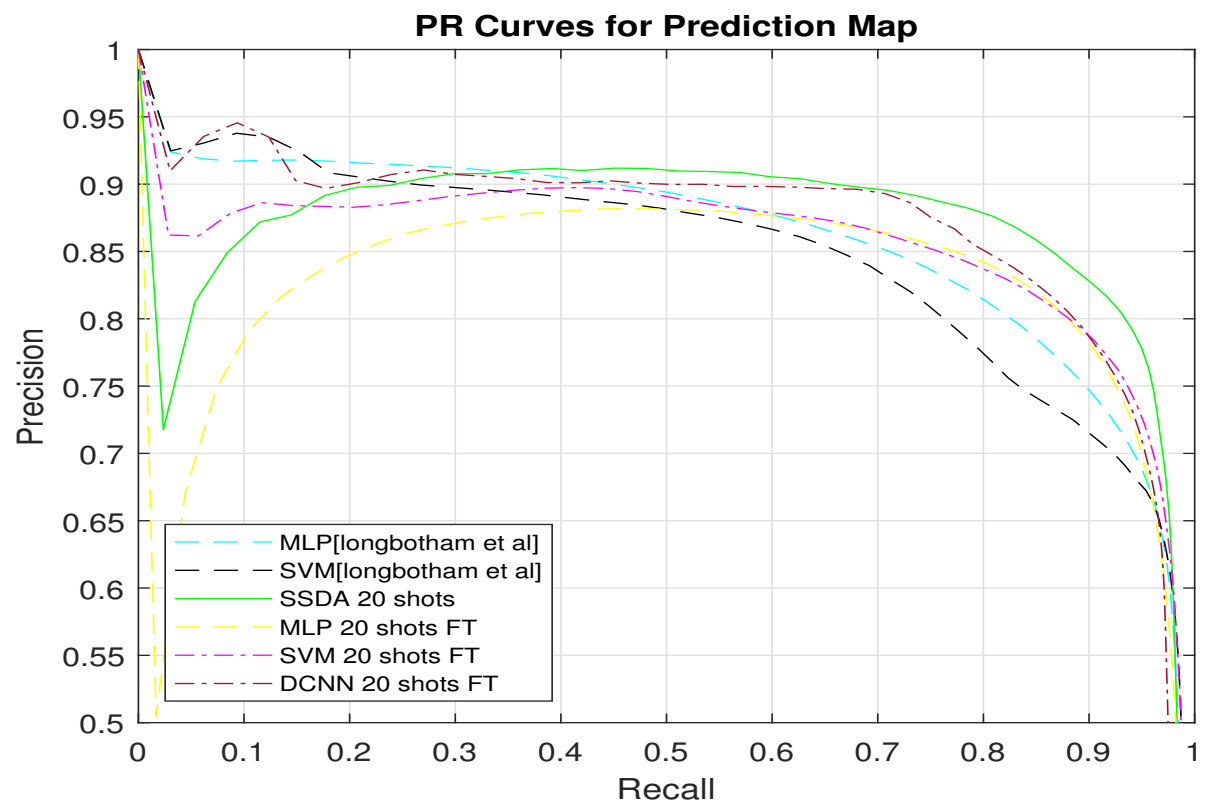

(a) Original Predicted Probability (OPP)

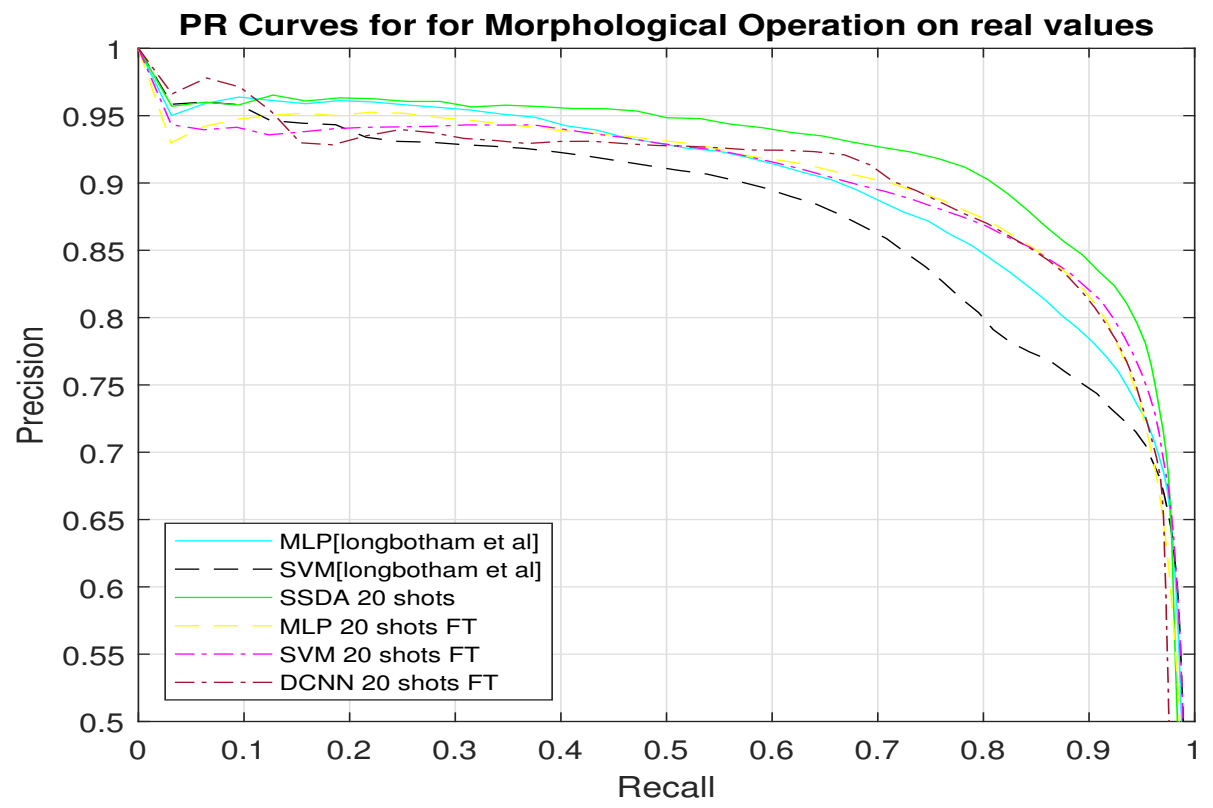

(b) After Morphological Operation with Disk Structure on OPP (MO-DS)

Figure 8. PR curves by different approaches on: (a) OPP map and (b) MO-DS map.

Results after Morphological Operation with Disk Structure on OPP (MO-DS)

To remove the false positive points from the OPP maps, we applied morphological operation on the maps with a structuring element size of 5 . The classification maps for different models are shown in Figure 9 and performance metrics shown as 'MO-DS' are listed in Table 1. Similarly, we display the probability maps using 'jet' colormaps where blue represents the probability ' 0 ' whereas red represents the probability of ' 1 '. The PR curves for the last six flood-detection scenarios in Table 1 are shown in Figure 8 b. 


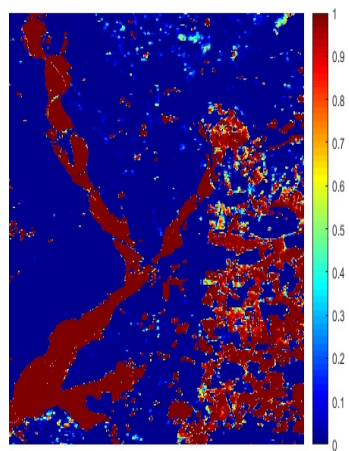

(a) CNN-SO

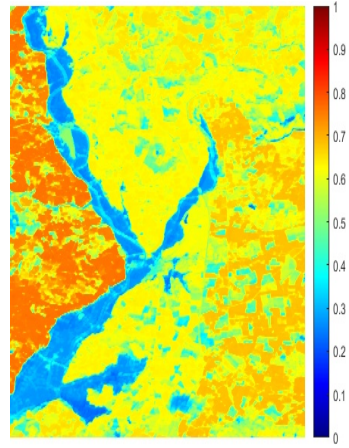

(e) SVM 1-shot FT

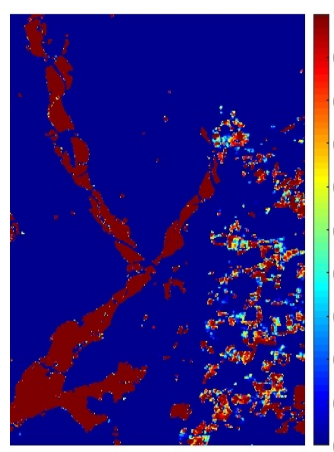

(i) DCNN 1-shot FT

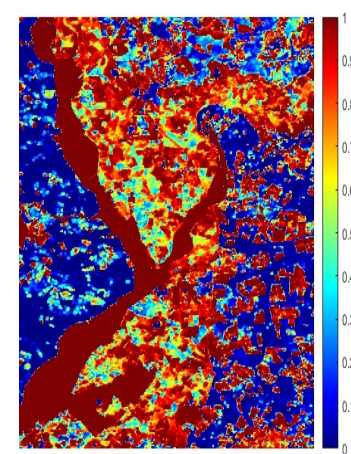

(b) SVM-SO

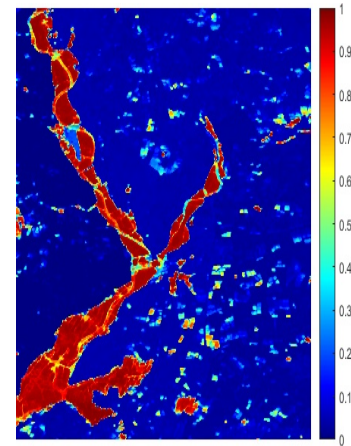

(f) SVM 20-shot FT

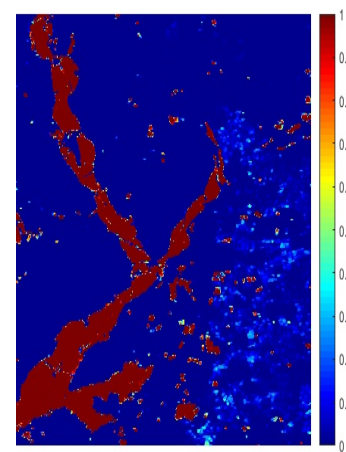

(j) DCNN 20-shot FT

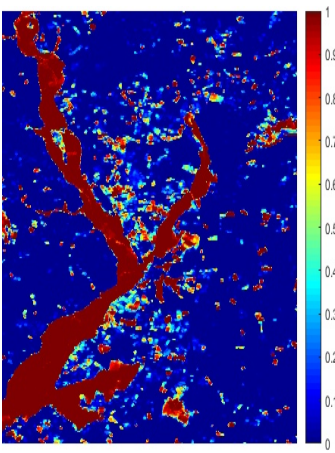

(m) SVM [8]

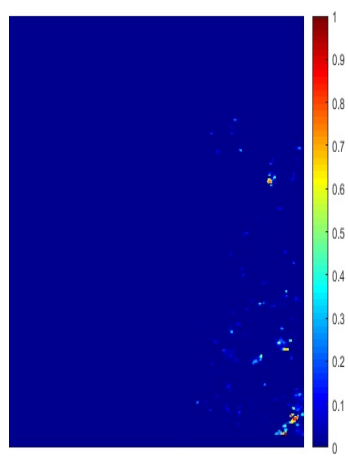

(c) MLP SO

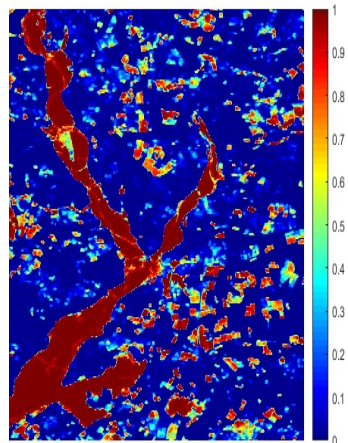

(g) MLP 1-shot FT

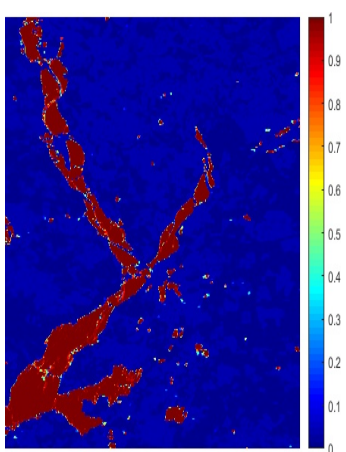

(k) SSDA 1-shot

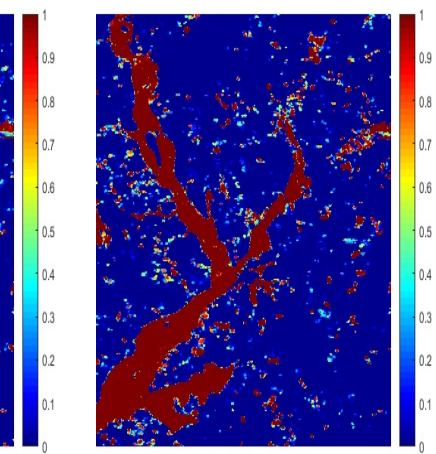

(n) MLP [8]

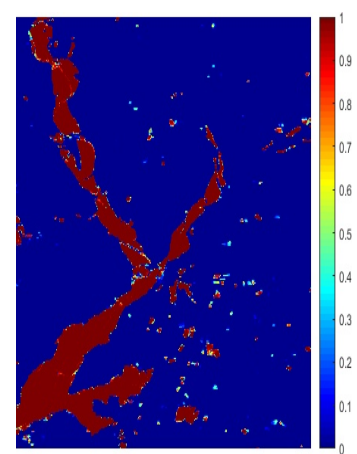

(d) UDA

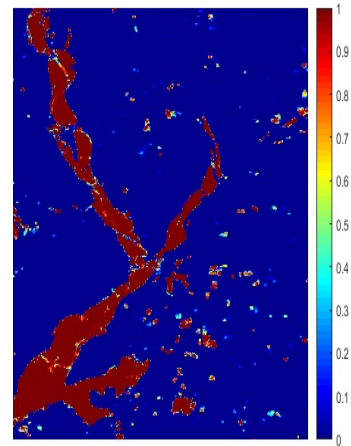

(h) MLP 20-shot FT

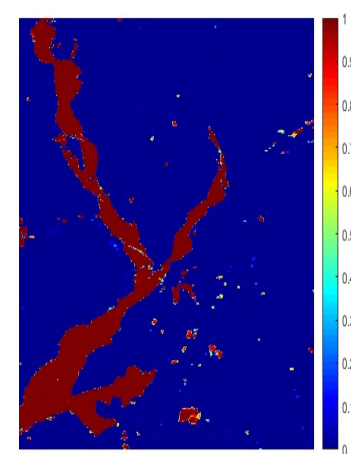

(1) SSDA 20-shot

Figure 9. Output after morphological operation with disk structures (MO-DS) on the OPPs. Blue represents the probability ' 0 ' and Red represents probability ' 1 '. 


\section{Discussions}

First, we compare the source only models in Table 1. In this setting, the CNN-SO method achieved the best F1 score of 0.5638 as compared with MLP-SO and SVM-SO (0.3466). For PR-AUC metric, CNN-SO (0.8070) and SVM-SO (0.8132) showed similar outcomes. The MLP-SO method failed to detect any flood samples in the post-event images. The UDA method, which used only unlabeled samples from the target domain performed better than all the source only models. It achieved similar results (F1 score of 0.8652 and PR-AUC of 0.8833 ) as compared to other few-shot methods which used a few labeled samples from the post-event images. For this dataset, UDA performed superbly without any labeled samples from target domain and the predicted flooding map is shown in Figure 9d. If we compare the UDA performance with the ground truth shown in Figure 2b, we can see that UDA produced very few false positives. The false positives were reduced by the post-processing step as shown in Figure 7d (before) and in Figure 9d (after) the processing step.

Next, we compare the performance of the SSDA method with the SVM, MLP and DCNN models with fine-tuning as described in Section 2.3.6. We can observe that all the methods performed similarly except that the SVM 1 shot FT method failed the task with Recall below 1\% (Table 1). The classification prediction maps shown in Figures $7 \mathrm{e}$ and $9 \mathrm{e}$ justify the results where the flood regions show close to ' 0 ' probabilities. In addition, SSDA methods are slightly better than the other three methods. In terms of PR-AUC and F1 score, SSDA achieved the highest scores three times and four times, respectively, as shown in the prediction maps (Figures 7 and 9). If we analyze the fine-tuning results by SVM, MLP and DCNN in Figures 7 and 9, MLP and DCNN performed similarly where the SVM fine-tuning with 1-shot was much worse. With 20 shots of labeled samples, SVM improved by a large margin as shown in Figure $9 \mathrm{f}$ as compared with the 1-shot result shown in Figure 9e. In Table 1, all the methods improved with increased number of shots. Please note that the improvements by all the methods from 10-shot to 20-shot are marginal. Therefore, we chose 20 labeled samples from the post-event images for fine-tuning and all the fine-tuning methods produced stable performances in the post-event images.

Flooding map predicted by the SVM classifier [8] had many false positives as shown in Figure 7m. After the morphological operation post-processing step, false positives were reduced by a large margin as shown in Figure $9 \mathrm{~m}$. The competition winner, the MLP classifier [8], showed similar performances. If we compare the PR-AUC metrics in Table 1, SSDA 20-shot achieved the best PR-AUC values after post-processing whereas SSDA 3-shot achieved the best PR-AUC value in original predicted probability map. After applying the morphological operation on the model predicted flood map in Figure 8a, all approaches improved their detection performances as shown in Figure 8b. In Figure 8a,b, SSDA 20-shot achieved the best performances.

It is worth noting that both the SVM and MLP classifiers used 48,684 labeled samples from the preand post-events images, respectively, for training. On the other hand, the domain adaptation method, SSDA, only used the 48,684 labeled samples from the pre-event images for training, and a few labeled samples in the post-event images for adaptation. Though the competition winner methods used more samples for training, these methods performed slightly worse than the SSDA 20-shot approach ( 0.8727 (SVM) and 0.8949 (MLP) vs. 0.9173). In addition, the SVM and MLP models [8] had a high chance to fail if the pre- and post-event images were not registered. The MLP and SVM with 20 samples described in Section 2.3.7 performed poorly as compared with the other methods in Table 1. Our experiments suggested that domain adaptation-based method, SSDA, is preferred for flood detection.

\section{Conclusions}

In this paper, we conducted a comparative study of various methods for flood detection including SVM, MLP, deep CNN models and domain adaptation method combined with those models. The comparison was conducted on the multi-temporal optical (Spot-5) and radar (SAR) sensor image dataset used for the flood-detection competition organized by DFTC in 2009. We found that all the compared methods performed similarly in flood detection, and the SSDA method, which is a combination of deep CNN with a semi-supervised domain adaptation strategy, achieved slightly 
better performance using much less training samples as compared with the competition winner methods. Our study suggests that the SSDA method is an effective algorithm for flood detection and it is preferred in practice. In future work, we will apply domain adaptation methods to more datasets across different locations to further validate our findings.

Author Contributions: Conceptualization, J.L., C.K.; methodology, K.A.I., M.S.U., C.K., J.L.; validation, K.A.I., M.S.U., C.K., J.L.; formal analysis, K.A.I., J.L., M.S.U., C.K.; investigation, K.A.I., M.S.U, C.K., J.L.; writing-original draft preparation, K.A.I., J.L.; writing-review and editing, K.A.I., J.L.; supervision, J.L., C.K.; project administration, J.L., C.K.; funding acquisition, J.L., C.K. All authors have read and agreed to the published version of the manuscript.

Funding: This research was developed with funding from the Defense Advanced Research Projects Agency (DARPA) under contract 140D6318C0043. The views, opinions and/or findings expressed are those of the author and should not be interpreted as representing the official views or policies of the Department of Defense or the U.S. Government.

Acknowledgments: The support of NVIDIA Corporation for the donation of the TESLA K40 GPU used in this research is gratefully acknowledged.

Conflicts of Interest: The authors declare no conflict of interest.

\section{References}

1. Guha-Sapir, D.; Vos, F.; Below, R.; Ponserre, S. Annual Disaster Statistical Review 2014: The Numbers and Trends. 2015. Available online: https://reliefweb.int/report/world/annual-disaster-statistical-review2014-numbers-and-trends (accessed on 28 July 2020).

2. Jonkman, S. Loss of life due to floods: General overview. In Drowning; Springer: Berlin, Germany, 2014; pp. 957-965.

3. Ashley, S.T.; Ashley, W.S. Flood fatalities in the United States. J. Appl. Meteorol. Climatol. 2008, 47, 805-818. [CrossRef]

4. Malinowski, R.; Groom, G.; Schwanghart, W.; Heckrath, G. Detection and delineation of localized flooding from WorldView-2 multispectral data. Remote Sens. 2015, 7, 14853-14875. [CrossRef]

5. Wang, Y. Using Landsat 7 TM data acquired days after a flood event to delineate the maximum flood extent on a coastal floodplain. Int. J. Remote Sens. 2004, 25, 959-974. [CrossRef]

6. Ireland, G.; Volpi, M.; Petropoulos, G.P. Examining the capability of supervised machine learning classifiers in extracting flooded areas from Landsat TM imagery: A case study from a Mediterranean flood. Remote Sens. 2015, 7, 3372-3399. [CrossRef]

7. Ahmed, M.R.; Rahaman, K.R.; Kok, A.; Hassan, Q.K. Remote sensing-based quantification of the impact of flash flooding on the rice production: A case study over Northeastern Bangladesh. Sensors 2017, 17, 2347. [CrossRef]

8. Longbotham, N.; Pacifici, F.; Glenn, T.; Zare, A.; Volpi, M.; Tuia, D.; Christophe, E.; Michel, J.; Inglada, J.; Chanussot, J.; et al. Multi-modal change detection, application to the detection of flooded areas: Outcome of the 2009-2010 data fusion contest. IEEE J. Sel. Top Appl. Earth Obs. Remote Sens. 2012, 5, 331-342. [CrossRef]

9. Twele, A.; Cao, W.; Plank, S.; Martinis, S. Sentinel-1-based flood mapping: A fully automated processing chain. Int. J. Remote Sens. 2016, 37, 2990-3004. [CrossRef]

10. Sun, D.; Yu, Y.; Zhang, R.; Li, S.; Goldberg, M.D. Towards operational automatic flood detection using EOS/MODIS data. Photogramm. Eng. Remote Sens. 2012, 78, 637-646. [CrossRef]

11. Mason, D.C.; Giustarini, L.; Garcia-Pintado, J.; Cloke, H.L. Detection of flooded urban areas in high resolution Synthetic Aperture Radar images using double scattering. Int. J. Appl. Earth Obs. Geoinf. 2014, 28, 150-159. [CrossRef]

12. Chowdhury, E.H.; Hassan, Q.K. Use of remote sensing data in comprehending an extremely unusual flooding event over southwest Bangladesh. Nat. Hazards 2017, 88, 1805-1823. [CrossRef]

13. Hong Quang, N.; Tuan, V.A.; Le Hang, T.T.; Manh Hung, N.; Thi Dieu, D.; Duc Anh, N.; Hackney, C.R. Hydrological/Hydraulic Modeling-Based Thresholding of Multi SAR Remote Sensing Data for Flood Monitoring in Regions of the Vietnamese Lower Mekong River Basin. Water 2020, 12, 71. [CrossRef]

14. Sun, D.; Yu, Y.; Goldberg, M.D. Deriving water fraction and flood maps from MODIS images using a decision tree approach. IEEE J. Sel. Top. Appl. Earth Obs. Remote Sens. 2011, 4, 814-825. [CrossRef] 
15. Liu, Z.; Li, G.; Mercier, G.; He, Y.; Pan, Q. Change detection in heterogenous remote sensing images via homogeneous pixel transformation. IEEE Trans. Image Process. 2017, 27, 1822-1834. [CrossRef] [PubMed]

16. Krizhevsky, A.; Sutskever, I.; Hinton, G.E. Imagenet classification with deep convolutional neural networks. In Advances in Neural Information Processing Systems; 2012; pp. 1097-1105. Available online: https:/ / papers. nips.cc/ paper / 4824-imagenet-classification-with-deep-convolutional-neural-networks.pdf (accessed on 28 July 2020).

17. Islam, K.A.; Pérez, D.; Hill, V.; Schaeffer, B.; Zimmerman, R.; Li, J. Seagrass detection in coastal water through deep capsule networks. In Proceedings of the Chinese Conference on Pattern Recognition and Computer Vision (PRCV), Guangzhou, China, 23-26 November 2018; pp. 320-331.

18. Islam, K.A.; Hill, V.; Schaeffer, B.; Zimmerman, R.; Li, J. Semi-supervised Adversarial Domain Adaptation for Seagrass Detection in Multispectral Images. In Proceedings of the 2019 IEEE International Conference on Data Mining (ICDM), Beijing, China, 8-11 November 2019; pp. 1120-1125.

19. Banerjee, D.; Islam, K.; Xue, K.; Mei, G.; Xiao, L.; Zhang, G.; Xu, R.; Lei, C.; Ji, S.; Li, J. A deep transfer learning approach for improved post-traumatic stress disorder diagnosis. Knowl. Inf. Syst. 2019, 60, 1693-1724. [CrossRef]

20. Islam, K.A.; Perez, D.; Li, J. A Transfer Learning Approach for the 2018 FEMH Voice Data Challenge. In Proceedings of the 2018 IEEE International Conference on Big Data (Big Data), Seattle, WA, USA, 10-13 December 2018; pp. 5252-5257.

21. Chowdhury, M.M.U.; Hammond, F.; Konowicz, G.; Xin, C.; Wu, H.; Li, J. A few-shot deep learning approach for improved intrusion detection. In Proceedings of the 2017 IEEE 8th Annual Ubiquitous Computing, Electronics and Mobile Communication Conference (UEMCON), New York, NY, USA, 19-21 October 2017; pp. 456-462.

22. Ning, R.; Wang, C.; Xin, C.; Li, J.; Wu, H. DeepMag+: Sniffing mobile apps in magnetic field through deep learning. Pervasive Mob. Comput. 2020, 61, 101106. [CrossRef]

23. Li, F.; Tran, L.; Thung, K.H.; Ji, S.; Shen, D.; Li, J. A robust deep model for improved classification of AD/MCI patients. IEEE J. Biomed. Health Inform. 2015, 19, 1610-1616. [CrossRef]

24. Kussul, N.; Lavreniuk, M.; Skakun, S.; Shelestov, A. Deep learning classification of land cover and crop types using remote sensing data. IEEE Geosci. Remote Sens. Lett. 2017, 14, 778-782. [CrossRef]

25. Li, W.; Fu, H.; Yu, L.; Cracknell, A. Deep learning based oil palm tree detection and counting for high-resolution remote sensing images. Remote Sens. 2017, 9, 22. [CrossRef]

26. Chi, J.; Walia, E.; Babyn, P.; Wang, J.; Groot, G.; Eramian, M. Thyroid nodule classification in ultrasound images by fine-tuning deep convolutional neural network. J. Digit. Imaging 2017, 30, 477-486. [CrossRef]

27. Jung, H.; Lee, S.; Yim, J.; Park, S.; Kim, J. Joint fine-tuning in deep neural networks for facial expression recognition. In Proceedings of the IEEE International Conference on Computer Vision, Las Condes, Chile, 11-15 December 2015; pp. 2983-2991.

28. Reyes, A.K.; Caicedo, J.C.; Camargo, J.E. Fine-tuning Deep Convolutional Networks for Plant Recognition. CLEF (Working Notes) 2015, 1391, 467-475.

29. Costache, R.; Ngo, P.T.T.; Bui, D.T. Novel Ensembles of Deep Learning Neural Network and Statistical Learning for Flash-Flood Susceptibility Mapping. Water 2020, 12, 1549. [CrossRef]

30. Jain, P.; Schoen-Phelan, B.; Ross, R. Automatic flood detection in SentineI-2 images using deep convolutional neural networks. In Proceedings of the 35th Annual ACM Symposium on Applied Computing, Brno, Czech Republic, 15 September 2020; pp. 617-623.

31. Nogueira, K.; Fadel, S.G.; Dourado, Í.C.; Werneck, R.d.O.; Muñoz, J.A.; Penatti, O.A.; Calumby, R.T.; Li, L.T.; dos Santos, J.A.; Torres, R.d.S. Exploiting ConvNet diversity for flooding identification. IEEE Geosci. Remote Sens. Lett. 2018, 15, 1446-1450. [CrossRef]

32. Sarker, C.; Mejias, L.; Maire, F.; Woodley, A. Flood mapping with convolutional neural networks using spatio-contextual pixel information. Remote Sens. 2019, 11, 2331. [CrossRef]

33. Gebrehiwot, A.; Hashemi-Beni, L.; Thompson, G.; Kordjamshidi, P.; Langan, T.E. Deep Convolutional Neural Network for Flood Extent Mapping Using Unmanned Aerial Vehicles Data. Sensors 2019, 19, 1486. [CrossRef]

34. Goodfellow, I.; Pouget-Abadie, J.; Mirza, M.; Xu, B.; Warde-Farley, D.; Ozair, S.; Courville, A.; Bengio, Y. Generative adversarial nets. In Proceedings of the Advances in Neural Information Processing Systems, Montreal, QC, Canada, 8-13 December 2014; pp. 2672-2680. 
35. Tzeng, E.; Hoffman, J.; Saenko, K.; Darrell, T. Adversarial discriminative domain adaptation. In Proceedings of the IEEE Conference on Computer Vision and Pattern Recognition, Honolulu, HI, USA, 21-26 July 2017; pp. 7167-7176.

36. Motiian, S.; Piccirilli, M.; Adjeroh, D.A.; Doretto, G. Unified deep supervised domain adaptation and generalization. In Proceedings of the IEEE International Conference on Computer Vision, Venice, Italy, 22-29 October 2017; pp. 5715-5725.

37. Rumelhart, D.E.; Hinton, G.E.; Williams, R.J. Learning representations by back-propagating errors. Nature 1986, 323, 533-536. [CrossRef]

38. Cortes, C.; Vapnik, V. Support-vector networks. Mach. Learn. 1995, 20, 273-297. [CrossRef]

39. Chang, C.C.; Lin, C.J. LIBSVM: A library for support vector machines. ACM Trans. Intell. Syst. Technol. 2011, 2, 1-27. [CrossRef]

40. Davis, J.; Goadrich, M. The relationship between Precision-Recall and ROC curves. In Proceedings of the 23rd International Conference on Machine Learning, Pittsburgh, PA, USA, 25-29 June 2006; pp. 233-240.

41. Saito, T.; Rehmsmeier, M. The precision-recall plot is more informative than the ROC plot when evaluating binary classifiers on imbalanced datasets. PLoS ONE 2015, 10, e0118432. [CrossRef]

42. Pedregosa, F.; Varoquaux, G.; Gramfort, A.; Michel, V.; Thirion, B.; Grisel, O.; Blondel, M.; Prettenhofer, P.; Weiss, R.; Dubourg, V.; et al. Scikit-learn: Machine Learning in Python. J. Mach. Learn. Res. 2011, $12,2825-2830$.

(C) 2020 by the authors. Licensee MDPI, Basel, Switzerland. This article is an open access article distributed under the terms and conditions of the Creative Commons Attribution (CC BY) license (http:/ / creativecommons.org/licenses/by/4.0/). 